Horizons philosophiques

\title{
La pensée politique de Jean Jaurès
}

\section{Paul Drouin}

Volume 5, numéro 2, printemps 1995

Le philosophe et l'état

URI : https://id.erudit.org/iderudit/800982ar

DOI : https://doi.org/10.7202/800982ar

Aller au sommaire du numéro

\section{Éditeur(s)}

Collège Édouard-Montpetit

\section{ISSN}

1181-9227 (imprimé)

1920-2954 (numérique)

Découvrir la revue

\section{Citer cet article}

Drouin, P. (1995). La pensée politique de Jean Jaurès. Horizons philosophiques, 5(2), 84-97. https://doi.org/10.7202/800982ar d'utilisation que vous pouvez consulter en ligne.

https://apropos.erudit.org/fr/usagers/politique-dutilisation/ 


\title{
LA PENSÉE POLITIQUE DE JEAN JAURĖS
}

\author{
"L'âme, pour réjouir une autre âme, emprunte à l'être \\ cettetransparence infinie etdouce qu'onappellela clarté'n. \\ Jean Jaurès
}

À une époque où il est facile d'oublier que socialisme rime avec humanisme, la pensée de Jean Jaurès (1859-1914) ne peut nous laisser indifférents. Elle nous aide à identifier les valeurs essentielles de l'État démocratique moderne et elle nous permet d'étudier les présupposés moraux du mouvement socialiste, lequel demeure, quoi qu'on en dise, un courant politique majeur pour saisir l'évolution du monde contemporain. La théorie de l'État de Jaurès fait de la justice le critère ultime de la philosophie, sa synthèse actualise la doctrine de l'école du droit naturel dont Grotius et Pufendorf sont les principaux artisans. La philosophie politique socialiste continue d'être un choix possible pour contrer l'idéologie néolibérale, elle peut apporter les correctifs indispensables pour réformer le système aveugle de la "croissance» capitaliste.

\section{La République démocratique}

La pensée de Jaurès au sujet de l'État s'est développée alors même que la démocratie politique amorçait son essor, lequel est loin d'être terminé comme les événements politiques du XXe siècle l'ont confirmé amplement. Dans son esprit, l'État démocratique revêt un caractère tantôt négatif, tantôt positif, selon la classe sociale qui en contrôle les leviers. Dans le cas où la bourgeoisie domine entièrement le gouvernement, l'État exerce plus aisément un pouvoir de répression à l'égard des travailleurs. Mais dès que ceux-ci mettent sur pied des organismes autonomes comme les syndicats et les coopératives qui leur permettent de développer l'apprentissage de la gestion, dès qu'ils coordonnent leurs efforts à l'intérieur d'un parti politique indépendant à l'égard du patronat, le chemin du pou-

1. Jaurès, Jean, De la réalité du monde sensible, Paris, Ed. Félix Alcan, 1891, p. 76. 
voir leur est ouvert par le suffrage universel, avec pour corollaire la réalisation de réformes qui pourront conférer à l'État démocratique un caractère positif. Depuis le congrès de la Deuxième Internationale tenu à Paris en 1900, les socialistes ont compris que le suffrage universel visant la conquête des municipalités et des assemblées nationales est tout à fait adapté à la période actuelle et que la formation d'une majorité de gauche, soutenant un gouvernement de gauche, demeure l'outil nécessaire de l'organisme législatif et gouvernemental pouvant conduire à l'égalité sociale.

L'État ne doit pas être vu comme étant nécessairement mauvais en soi car, dans l'État de type républicain qui est une conquête hautement appréciable, l'égalité des droits est donnée aux citoyens par le suffrage universel qui fonde la souveraineté politique. Dans ces conditions, le problème de la nature de l'État se ramène aux orientations qui lui sont données par les classes qui luttent entre elles pour gagner l'hégémonie politique et économique de la société. De même, on ne peut résumer le contenu de l'État par une seule classe en disant, par exemple, qu'il est un État bourgeois. Cette formule est une négation du rapportévolutif des forces sociales, elle nie la lutte des travailleurs contre la classe possédante et elle ignore la réalité objective du mouvement social qui est partie intégrante de la nature évolutive des choses. «Figer l'État, c'est supprimer l'espérance, c'est supprimer l'action. Non, l'État démocratique d'aujourd'hui n'est pas un bloc homogène et d'un seul métal ${ }^{2}$ ". Le gouvernement de l'État moderne n'est pas uniquement une organisation de domination de classe. L'État d'aujourd'hui est plutôtl'expression de la démocratie politique au sein de laquelle la puissance des travailleurs peut grandir. C'est le rapport des forces sociales qui détermine la nature de l'État. L'existence combinée de la lutte des classes puis de la collaboration entre les classes est une donnée régulatrice pour comprendre l'évolution du mouvement social. L'État n'est donc pas l'expression d'une seule classe, même si celle-ci domine économiquement et politiquement.

2. Jaurès, J., L'Armée nouvelle, introduction de Louis Baillot, Paris, Editions sociales, 1978, p.316 
«En fait, l'État n'exprime pas une classe, il exprime le rapport des classes, je veux dire le rapport de leurs forces ${ }^{3}$ ".

Jaurès privilégie la république parce qu'elle est une forme étatique supérieure à la monarchie et qu'elle facilite un meilleur fonctionnement de la démocratie politique. La république est une structure institutionnelle qui convient mieux à l'État démocratique parce que, dans la république, forme nécessaire du droit, les citoyens peuvent «tracer eux-mêmes la règle commune de leur action ${ }^{4} »$. La république annonce et prépare le socialisme, "elle le contient même déjà en quelque mesure, puisque seule elle y peut conduire par une évolution légale, sans rupture de continuité ${ }^{\prime \prime}$. En tant que forme d'organisation, la république politique favorise la dignité pour toutes les personnes de la nation; elle est du reste devenue un modèle qui a inspiré les régimes démocratiques à travers le monde. La république politique est née en 1789 avec la proclamation souveraine des droits fondamentaux qui a donné à tout individu, indépendamment de ses origines sociales, la même dignité et les mêmes droits. Avec la Révolution française, les obstacles formels à la souveraineté du peuple ont été levés pour faire en sorte que tous les individus deviennent des rois, que toutes les personnes deviennent égales en droit, la loi devenant l'expression de la volonté générale.

Mais la liberté, l'égalité et la justice n'entrent pas uniquement en ligne de compte dans les relations politiques, elles doivent pénétrer aussi les rapports économiques, la production et le travail qu'il est possible d'organiser selon un modèle républicain. La république politique jette les bases de l'édification de la république sociale, mais il subsiste, dans cette forme d'organisation, une contradiction entre l'ordre politique etl'ordre économique. Les partis politiques de la bourgeoisie désertent la politique républicaine en n'acceptant pas que la nation soit

3. Ibid. p.315.

4. Jaurès, J., "Discours à la jeunessen, in L'esprit du socialisme, Six discours et études, préface de Jean Rabaut, Paris, Ed. Gonthler, Coll. Médiations, 1964, p.56.

5. Jaurès, J., Etudes socialistes, présentation de Madeleine Rebérioux, GenèveParis, Ed. Slatkine Reprints, Coll. Ressources, 1979, pp. LXIII-LXIV. 
souveraine dans l'ordre économique comme elle est souveraine dans l'ordre politique: "Oui, par le suffrage universel, par la souveraineté nationale, qui trouve son expression définitive et logique dans la République, vous avez fait de tous les citoyens, y compris les salariés, une assemblée de rois. C'est d'eux, c'est de leur volonté souveraine qu'émanent les lois et le gouvernement; ils révoquent, ils changent leurs mandataires, les législateurs et les ministres; mais, au moment même où le salarié est souverain dans l'ordre politique, il est dans l'ordre économique réduit à une sorte de servage ${ }^{6}$ ".

\section{Souveraineté et laïcité}

La démocratie est avant tout l'égalité des droits et la souveraineté politique du peuple. Dans les nations modernes, l'exercice de la souveraineté ne peut être subordonné à aucune formule dogmatique. "Il suffit qu'il y ait des citoyens, il suffit qu'il $y$ ait des êtres majeurs ayant leur liberté, leur personnalité et désireux de mettre en œuvre ce droit pour que la nation moderne dise : Voilà la source unique et profonde de la souveraineté ${ }^{7}$ ". Depuis la Révolution française, la conscience libre, la liberté individuelle réglée par le devoir est à la base de la souveraineté politique. Dans l'ordre politique, les socialistes doivent lutter pour le développement de la démocratie. Et la république est l'organisation politique qui incarne le mieux l'idéal démocratique puisqu'elle repose sur la souveraineté pleine et entière du peuple. Jaurès souligne que le mouvement démocratique s'est développé dans le monde en même temps que la philosophie et l'action socialistes. II insiste sur un point : pour que surgisse le socialisme, il a fallu que l'idée de démocratie s'affirme complètement. Et la démocratie ne peut se développer que par la reconnaissance du droit de la personne à la liberté. Ce droit ne doit être ni relatif ni précaire, mais absolu et définitif, carc'est par l'affirmation de la liberté que tous les êtres humains peuvent devenir égaux entre eux.

6. Jaurès, J., "République et socialisme», in Pages choisies, introduction de Paul Desanges et Luc Mériga, Paris, Ed. Rieder, 1922, p.321.

7. Jaurès, J., "Pour la laîque», in L'esprit du socialisme, op.cit., p.128. 
La liberté est la base véritable de l'égalité et de la solidarité. La connexion nécessaire entre la liberté et l'égalité définit la laïcité et le droit. La liberté individuelle peut s'exercer pourvu que l'égalité des droits puisse être assurée par l'État dans un régime démocratique. L'égalité favorise la liberté et cette dernière donne l'impulsion morale - l'autonomie de la volonté inhérente à la liberté - qui déclenche la réalisation des conditions de l'égalité. Lajustice peut être concrétisée socialement sil'équilibre est trouvé sur la base de l'égalité. Les mécanismes et les mesures légales protégeant cet équilibre sont d'inspiration laïque et la justice ne peut être atteinte que si les lois votées par les gouvernements le sont dans l'esprit de la laïcité. Dans une république démocratique, toute personne, peu importe sa religion, a le droit de participer à la souveraineté. C'est dire que la religion ne peut être à la base de l'éducation puisqu'elle n'est pas à la base de la souveraineté.

Aujourd'hui, une éthique qui, au nom du modernisme ou du postmodernisme, négligerait ou évacuerait la laïcité de l'État et de l'école serait incapable de contrer l'intégrisme et le fanatisme religieux qui menacent concrètement les droits et libertés démocratiques à l'échelle internationale. On n'a qu'à penser à Salman Rushdie ou à Taslima Nasreen pour se représenter à quel point la laïcité des institutions civiles demeure une exigence de base essentielle pour garantir le respect de la liberté de conscience. Les représentants de la religion ne doivent pas se mêler des affaires temporelles mais s'occuper uniquement de la dimension spirituelle. La laïcité estl'âme de la république, elle se confond avec l'application des principes démocratiques de liberté et d'égalité. Puisant dans l'école du droit naturel et dans la philosophie des Lumières du XVIIle siècle, Jaurès est convaincu que l'instruction publique estun grand levier pour éveiller la conscience de l'humanité et qu'une plus grande égalité des citoyens peut rendre encore plus intenable l'inégalité sociale. La laïcité est indissociable de la liberté de conscience, elle se définit précisément par le respect de la liberté de conscience qui est elle-même la liberté des libertés dans une société démocratique. Et la liberté est le droit inné à l'entière réalisation, 
en tout être humain, de la dignité humaine. Mais une simple déclaration de principe ne suffit pas.

L'État souverain, l'État de droit doit assurer des garanties réelles afin que chaque être humain puisse se développer comme une personne libre. Jaurès pense comme Hegel que l'État doit placer la liberté individuelle au centre des lois. II précise que la liberté de conscience ne peut s'exercer que lorsque la raison est vraiment libre. Comprimer ou restreindre la raison, c'est asservir la liberté. Ce serait trahir la raison et la liberté que de confier l'enseignement public à une Église. En développant l'exercice de la faculté de juger par la raison, les enfants qui fréquentent l'école laïque peuvent cultiver à la fois le sentiment de la dignité humaine et le goût de la liberté, sans lesquelles l'humanité n'existerait pas. La liberté de l'esprit est ce qu'il y a de plus précieux au monde. Aucun dogme ne doit limiter la recherche de l'humanité qui est une commission d'enquête dans l'univers.

Pour favoriser l'universalisation du droit naturel à la liberté de conscience, il est absolument nécessaire qu'aucune Eglise n'ait le monopole de la conscience des jeunes dans les écoles publiques. La séparation de l'Église et de l'école constitue à la fois la condition et la conséquence du respect de la liberté de conscience. Chaque personne, chaque Eglise peut croire et penser ce qu'elle veut, pourvu qu'elle n'impose pas ses croyances aux autres. En votant la Loi de la séparation de l'Église et de l'État en 1905, la Chambre des députés de la République française voulait faire de la liberté de conscience une réalité vivante et non seulement un principe déconnecté. En adoptant cette loi, les républicains et les socialistes appuyaient, en fait, une loi de laïcisation de l'Etat qui est une loi de liberté, une loi grâce à laquelle les enfants peuvent apprendre à vivre les uns avec les autres dans la même liberté sans qu'aucun culte ne soit privilégié par l'Etat laïque et la nation.

L'école républicaine que voulait instaurer Condorcet est en fin de compte l'école laïque. Fondés sur la raison, les principes moraux n'appartiennent pas à une seule religion mais plutôt à toute la collectivité. Reconnaissant à toutes les personnes la 
liberté de conscience et d'opinion en matière religieuse, la Déclaration des droits de 1789 ne permet pas de détruire l'égalité en accordant des privilèges ou des avantages à une doctrine religieuse particulière. Il est nécessaire de séparer la morale de la religion dans l'instruction publique et de n'enseigner les dogmes d'aucune religion. En toute liberté, les parents peuvent envoyer leurs enfants dans les temples de leur choix pour qu'ils reçoivent des leçons religieuses, mais l'immense majorité qui décide d'envoyer ses enfants dans les établissements scolaires nationaux doitêtre assurée du respect pour tous des droits fondamentaux à l'égalité et à la liberté de conscience. La souveraineté a été érigée sur une base laïque etl'éducation, qui est une institution essentielle de la république, doit également être constituée sur une base civile. Indépendamment de tout système religieux, la démocratie a fondé le droit en ne comptant que sur l'égale dignité des êtres humains et elle doit être, pour cette raison, laïque dans sa morale comme dans ses institutions.

La laïcité et la démocratie ne peuvent être dissociées sans porter préjudice aux citoyens car "qui dit obligation, qui dit loi, ditnécessairement laïcité ${ }^{\prime}$ ". Or la première obligation, le premier devoir imposé par la loi à l'enfant est d'aller à l'école et si, dès son plus jeune âge, il est éduqué dans la confusion du pouvoir spirituel et du pouvoir temporel, comment fera-t-il plus tard pour établir «la distinction nécessaire entre l'ordre religieux, qui ne relève que de la conscience individuelle, et l'ordre social et légal qui est essentiellement laïque ${ }^{9}$. Nous n'avons pas besoin d'inventer une éthique laïque puisqu'elle existe depuis la Révolution française. Elle est même devenue un fait social favorisant l'unanimité morale qui a contribué à diminuer la violence aveugle et qui a aidé au développement d'un progrès pacifique et légal. La démocratie ne saurait permettre à des principes contraires de s'installer dans les institutions civiles et dans l'école publique.

\footnotetext{
8. Jaurès, J., "L'éducation laĩque", in Jean Jaurès - Bulletin de la Société d'Etudes Jaurésiennes, No.26, juillet-septembre 1967, p.8.

9. Ibid., p.8.
} 


\section{L'humanisme socialiste}

La culture laïque de la Révolution française a jeté les bases d'une éthique socialiste et humaniste qui fait de la réalisation de la justice sociale le but suprême à atteindre. "Nous voulons que l'égalité pénètre dans la vie des hommes ${ }^{10}$ ". Pour Jaurès comme pour Émile Durkheim, "le socialisme possède donc avant tout une origine morale ${ }^{11} »$. Réalisation de l'ordre moral dans la sphère politique, le socialisme doitêtre aussi la réalisation de l'ordre moral dans la sphère économique. Le capitalisme fait obstacle à la vie morale. Afin que celle-ci devienne possible, il est nécessaire de mettre fin à la contradiction entre la souveraineté politique du citoyen assurée par le suffrage universel et la dépendance économique du salarié dans le travail. La propriété a admis l'esclavage dans l'Antiquité et le servage au Moyen-Age. Aujourd'hui la propriété bourgeoise comporte le salariat. Rien ne laisse présager qu'elle ne se modifiera pas encore puisque la forme capitaliste de la propriété divise la société en classes antagonistes, le travail n'appartient pas vraiment à ceux qui produisent, "le droit humain en eux est donc incomplet et mutilé ${ }^{12}$ ».

Continuateur de Fichte, Proudhon et Marx, Jaurès constate que le capital est attribué à une minorité possédante et que cette injustice fondamentale porte atteinte à la liberté des travailleurs tout en entraînant l'aliénation de la personne. Les richesses accumulées et les moyens de production créés par la communauté devraient pourtant être à sa disposition et contribuer à son affranchissement. "Et comme la communauté ne peut assurer le droit de l'individu qu'en mettant à sa disposition les moyens de produire, il faut que la communauté elle-même soit investie, sur ces moyens de produire, d'un droit souverain de propriété ${ }^{13}$ ". Le seul cadre permettant qu'aucune personne ne soit écartée de la sphère du droit, c'est que les responsabilités

10. Jaurès, J., "Civilisation et socialisme", in Clarté, No.40, 31 juillet 1923, p.336.

11. Birnbaum, P., "Préface», in Durkheim, Emile, Le socialisme, Paris, Presses Universitaires de France, Coll. Quadrige, 1992, p.16.

12. Jaurès, J., "Le but", Études socialistes, op.cit., p.126.

13. Jaurès, J., "Le socialisme et la vie», Etudes socialistes, op.cit., p.138. 
économiques soient de plus en plus assumées par la communauté nationale. Chaque nation a sa physionomie et son génie propres. Fondamentalement, «l'idée nationale et l'idée socialiste sont liées ${ }^{14}$ ". La solidarité organisée de la nation peut favoriser l'accession à la propriété et, par conséquent, la liberté pour tous. La communauté nationale doit entrer dans la sphère d'action du pouvoir, seule garantie pour qu'il y ait équilibre entre la solidarité et la liberté. "Nous touchons ici à un des plus délicats problèmes, non seulement de l'ordre socialiste, mais de l'ordre humain ${ }^{15}$ ". L'égalité politique ne peut suffire, il faut également assurer l'égalité économique et sociale. La nation doit être l'organe du progrès social. Sur la base de la propriété collective, tous les citoyens de la nation peuvent devenir copropriétaires en déléguant leur droit de propriété à des responsables élus, mandatés et révocables. La condition de la liberté véritable, c'est l'accessibilité pour tous à la propriété et celle-ci est facilitée par la socialisation ou la nationalisation de la propriété afin qu'elle devienne collective. Le socialisme veut développer la propriété pour toutes les personnes. Ce changement social en profondeur ne peut faire que du bien à tous. Les travailleurs comme les bourgeois doivent devenir copropriétaires de la puissance économique. Nous nous acheminerons progressivementainsivers la disparition complète des classes sociales, la suprématie d'une classe sur une autre étant "un attentat à l'humanité ${ }^{16}$ ".

Certes, la répression consécutive à la Révolution française a montré que les droits humains doivent être réalisés par des voies meilleures, c'est-à-dire par des voies libres et démocratiques. En ce sens, la conception socialiste de Jaurès refuse le pouvoir illimité de l'État. Pour lui, ce ne doit pas être uniquementl'État central qui organise la production mais la libre spontanéité décentralisée des individus et des groupes locaux de travailleurs qui doivent avoir la plus largè autonomie. L'ordre

14. Jaurès, J., «En Sorbonne», in Oeuvres, textes présentés par Max Bonnafous, Paris, Ed. Rieder, 1931-1939, Vol.3, p.426.

15. Jaurès, J., L'organisation socialiste, in Oeuvres, Vol.3, op.cit., p.390.

16. Jaurès, J., "Le socialisme et la vie», Etudes socialistes, op.cit., p.135. 
économique ne sera pas dépendant de l'ordre politique si le système électif se substitue partout au régime autoritaire. Manifestement, "tout le socialisme, tout le collectivisme n'est pas dans Marx ${ }^{17}$ ". L'organisation du travail envisagée par Jaurès intègre notamment la critique du socialisme anarchiste puisque "si nous allons vers l'égalité et la justice, ce n'est pas aux dépens de la liberté : nous ne voulons pas enfermer les hommes dans des compartiments étroits, numérotés par la force publique. Nous ne sommes pas séduits par un idéal de réglementation tracassière et étouffante ${ }^{18^{\prime}}$.

Après la Révolution d'Octobre 1917, les léninistes, puis les staliniens, à des degrés variables, se sont largement écartés des voies de la liberté, de la démocratie et du socialisme laïque et humaniste. En URSS, quelles horreurs le "socialisme réel» n'a-t-il pas engendrées! Le socialisme doit étendre les espaces de liberté, non les restreindre, voire les anéantir par une dictature sanguinaire. Le socialisme doit s'appliquer à protéger et à promouvoir énergiquement les droits individuels. II faut tirer les leçons qui s'imposent du bureaucratisme policier au sein duquel les travailleurs, prétendument libérés des exploiteurs en URSS et dans les autres "démocraties populaires", se sont retrouvés dans l'universelle servitude. Jamais aucun argument objectif ne pourra justifier moralement le sacrifice de la liberté et des droits humains. "La justice sociale ne peut être séparée de la liberté ${ }^{19}$ ". Précisément parce qu'il était incompatible avec le mouvement d'expansion de la liberté, le "socialisme réel» devaits'effondrer. "Nous voulons qu'aucun homme dans l'usine ou aux champs ne soit l'outil d'un autre homme ${ }^{20}$ ". Jaurès rejoint Kant pour qui l'être humain doit toujours être considéré comme but et non comme moyen, impératif qui implique une société sans classes et l'élimination de toute servitude entre les hommes. Dans ce cadre éthique, l'humanisme socialiste vise

17. Jaurès, J., "Collectivisme», in Oeuvres, Vol.3, op.cit., p.159.

18. Jaurès, J., L'organisation socialiste, Vol. 3, op.cit., p.329.

19. Jaurès, J., cité par Droz, J., Le socialisme démocratique, Ed. Armand Colin, 1966, p.80.

20. Jaurès, J., "Socialisme et liberté", in Oeuvres, Vol.6, op.cit., p.77. 
une société égalitaire pour supprimer les iniquités de la société actuelle car "au fond du capitalisme, il y a la négation de l'homme ${ }^{21}$ ". Le socialisme que Jaurès préconisait reste donc à construire.

\section{La fraternité des nations}

Etl'idéal socialiste veut réaliser la réconciliation internationale par la combinaison des qualités et des facultés des différents peuples. En établissant la collaboration nationale, le socialisme mettra progressivement un terme à la lutte des classes et il pourra en finir aussi avec la lutte fratricide qui oppose les peuples entre eux à l'échelle internationale. La concorde et l'entraide peuvent s'établir non seulement entre les travailleurs d'une même nation mais aussi entre les différents peuples. " $\mathrm{Ce}$ n'est donc que par la libre fédération de nations autonomes répudiant les entreprises de la force et se soumettant à des règles de droit, que peut être réalisée l'unité humaine ${ }^{22}$ ". Jaurès et Edouard Vaillant ont mené une action énergique et résolue pour la paix en tant que dirigeants de la Deuxième Internationale. Cette question était prioritaire et ils définissaient la politique internationale du socialisme par l'action coordonnée en faveur de la paix.

Les socialistes doivent agir contre le militarisme et la guerre. Cette action n'est pas une déviation, au même titre que la politique socialiste déployée pour laïciser l'enseignement et l'État n'en est pas une. Dès 1907, l'Internationale socialiste proclamait la légitimité de l'existence nationale, le droit à l'indépendance et à l'inviolabilité des nationalités; elle affirmait en même temps «le devoir des prolétaires de tous les pays de s'organiser pour maintenir la paix ${ }^{23}$ ". Quand un litige divise deux peuples, l'arbitrage international est le moyen pour éviter la guerre. Souvent, les diplomates et les gouvernants ne

21. Jaurès, J., "Introduction à la morale sociale de Benoît Malon", in Oeuvres, Vol.3, op.cit., p.267.

22. Jaurès, J., L'Armée nouvelle, op. cit., p. 329-330.

23. Jaurès, J., "Le Congrès de Stuttgart et l'antimilitarisme", in Oeuvres, Vol.5, op.cit., p.124. 
s'empressent pas de réaliser l'arbitrage et préfèrent se défiler. C'est la volonté coordonnée des travailleurs de tous les pays qui devra imposer "l'arbitrage de l'humanité, l'arbitrage de la raison ${ }^{24}$ ". L'action pour éviter les conflits par l'arbitrage est aussi un moyen pour permettre la satisfaction des besoins sociaux plutôt que d'encourager la croissance absurde de budgets militaires accablants et destructeurs.

Jaurès a été assassiné le 31 juillet 1914 au Café du Croissant, rue Montmartre, dans le deuxième arrondissement, à Paris. Raoul Villain, le meurtrier, était un nationaliste catholique fanatisé par des journaux comme l'Action française et l'Echo de Parisqui, plus d'une fois, avaientappelé au châtiment exemplaire contre celui qu'ils considéraient comme un traître pangermaniste. La volonté des ultra-nationalistes et des industriels de la mort fut exaucée. La nouvelle se répandit aussitôt avec la clameur populaire qui disait : "lls ont tué Jaurès, c'est la guerre ${ }^{25}$ ". Le lendemain, $1^{\text {er }}$ août, la mobilisation générale était décrétée en France et en Allemagne. Pourquoi a-t-il été tué? Jaurès ne se résignait pas à accepter la boucherie des humains contre les humains. II n'a jamais cessé de dénoncer les scandales «du capitalisme de l'obus, du canon et de la mitrailleuse ${ }^{26}$ » en plus de réclamer une réforme de la fiscalité afin que les classes capitalistes assument le poids financier de leur politique guerrière. Dans les pays concernés par la menace d'un conflit, il voulait substituer l'arbitrage international à la guerre par le moyen de la grève générale simultanée des travailleurs concentrés surtout dans les industries d'armement. Son engagement risquait d'entretenir le mouvement social agissant pour stopper la perpétuation du désordre capitaliste oppresseur et meurtrier, il contrecarrait les plans des fauteurs de guerre, aussi bien dans les rangs des partis politiques de la bourgeoisie impérialiste que chez les dirigeants socialistes. On l'a donc assassiné. Jaurès avait confiance dans la grève générale mais

24. Ibid., p.132.

25. Rabaut, J., Jaurès assassiné, Bruxelles, Ed. Complexes, 1984, p.71.

26. Jaurès, J., cité par Haupt, Georges, Le congrès manqué, l'Internationale à la veille de la première guerre mondiale, Paris, Ed. François Maspero, 1965, p.61. 
il a sous-estimé l'opposition contre ce moyen d'action, aussi bien dans la social-démocratie allemande qu'au sein du mouvement socialiste en France. Après son assassinat, le chemin était libre pour appeler au ralliement de toutes les classes dans l'union sacrée pour la guerre. Dès lors, l'internationalisme de Jaurès était abandonné. Le jour même de ses funérailles, le 4 août, les députés socialistes votaient en faveur des crédits de guerre.

L'idéal socialiste de Jaurès se concrétisera graduellement si la souveraineté s'étend de plus en plus, si elle s'affirme partout comme un mode d'organisation pouvant conduire à la démocratie sociale. Si la démocratie politique républicaine continue d'être, à l'heure présente, un objectif essentiel à atteindre pour le développement des nations, celle-ci ne pourra s'étendre véritablement que par la laïcisation de l'Etat. La souveraineté, la démocratie politique ne sera viable que sur une base laïque et non-confessionnelle. L'instruction laïque est un moyen irremplaçable de progrès social dans la mesure où elle place au-dessus de tout le respect absolu de la liberté de conscience ${ }^{27}$. Cela est d'autant plus urgent, en cette fin de siècle, que montent les intégrismes, cousins du fascisme, qui promettent d'autres millions de morts. Ce n'est ni de dogmatisme ni de sectarisme dont nous avons besoin, mais d'un idéal chargé d'espérance universelle qui puisse être adapté aux circonstances historiques spécifiques. Les socialistes ne projettent pas cet idéal dans le vide. "lls ne peuvent le réaliser que dans la nation autonome, selon des méthodes d'action et de combat que suggère ou qu'impose l'histoire de chaque pays, avec les éléments fournis par chacune des substances nationales ${ }^{28}$ ".

La pensée de Jaurès nous aide à comprendre notre monde et à ouvrir des voies de solution. Elle peut même, ici et maintenant, contribuer au débat qui doit déterminer l'avenir

27. Le gouvernement du Québec doit «lever l'hypothèque confessionnelle qui étouffe l'école québécoise" (Daniel Baril, Les mensonges de l'école catholique, Montréal, VLB éditeur, Coll. Parti pris actuels, 1995, p. 182).

28. Jaurès, J., L'Armée nouvelle, op.cit., p.329. 
politique du Québec ${ }^{29}$. Affirmer la pleine souveraineté de la nation québécoise exige le dépassement des préjugés néolibéraux et conservateurs qui ne peuvent aboutir qu'à une souveraineté fragmentée, morcelée. La conquête de la liberté est inséparable du mouvement vers la solidarité nationale, vers la République sociale.

Paul Drouin Collège Edouard-Montpetit

29. Durant la campagne électorale qui a porté le Parti québécois au pouvoir en 1994, le chroniqueur Pierre Graveline, s'appuyant sur Jean Jaurès qui avançait «une conception plutôt élevée de la démocratie" ("Johnson, Parizeau et Jaurès", Le Devoir, 9 août 1994), rappelait aux partis en lice que c'est de la volonté souveraine de tous les citoyens, y compris les salariés, qu'émanent les lois et le gouvernement. A la veille de la consultation référendaire, il souhaitait vivement que le peuple québécois vote en faveur de la souveraineté puisqu'il possède tous les attributs d'une nation qui peut disposer librement d'elle-même en vertu du droit international («L'obligation morale de faire la souveraineté", Le Devoir, 26 janvier 1995). 